\title{
Quantized field description of rotor frequency-driven dipolar recoupling
}

Cite as: J. Chem. Phys. 112, 1096 (2000); https://doi.org/10.1063/1.480664

Submitted: 25 August 1999 . Accepted: 22 October 1999 . Published Online: 06 January 2000

G. J. Boender, S. Vega, and H. J. M. de Groot

\section{ARTICLES YOU MAY BE INTERESTED IN}

Chemical shift correlation spectroscopy in rotating solids: Radio frequency-driven dipolar recoupling and longitudinal exchange

The Journal of Chemical Physics 96, 8624 (1992); https://doi.org/10.1063/1.462267

Homonuclear radio frequency-driven recoupling in rotating solids

The Journal of Chemical Physics 108, 9463 (1998); https://doi.org/10.1063/1.476420

${ }_{13 C}-{ }^{13} \mathrm{C}$ dipolar recoupling under very fast magic angle spinning in solid-state nuclear

magnetic resonance: Applications to distance measurements, spectral assignments, and highthroughput secondary-structure determination

The Journal of Chemical Physics 114, 8473 (2001); https://doi.org/10.1063/1.1359445

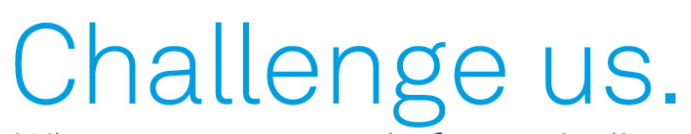

What are your needs for periodic

signal detection?

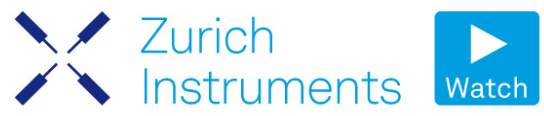

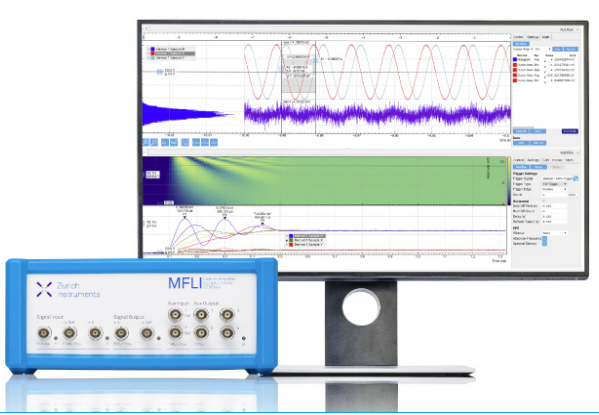




\title{
Quantized field description of rotor frequency-driven dipolar recoupling
}

\author{
G. J. Boender ${ }^{\mathrm{a})}$ \\ Leiden Institute of Chemistry, Leiden University 2300 RA Leiden, The Netherlands \\ and Department of Chemical Physics, Weizmann Institute of Science, 76100 Rehovot, Israel \\ S. Vega \\ Department of Chemical Physics, Weizmann Institute of Science, 76100 Rehovot, Israel \\ H. J. M. de Groot \\ Leiden Institute of Chemistry, Leiden University 2300 RA Leiden, The Netherlands
}

(Received 25 August 1999; accepted 22 October 1999)

A formalized many-particle nonrelativistic classical quantized field interpretation of magic angle spinning (MAS) nuclear magnetic resonance (NMR) radio frequency-driven dipolar recoupling (RFDR) is presented. A distinction is made between the MAS spin Hamiltonian and the associated quantized field Hamiltonian. The interactions for a multispin system under MAS conditions are described in the rotor angle frame using quantum rotor dynamics. In this quasiclassical theoretical framework, the chemical shift, the dipolar interaction, and radio frequency terms of the Hamiltonian are derived. The effect of a generalized RFDR train of $\pi$ pulses on a coupled spin system is evaluated by creating a quantized field average dipolar-Hamiltonian formalism in the interaction frame of the chemical shift and the sample spinning. This derivation shows the analogy between the Hamiltonian in the quantized field and the normal rotating frame representation. The magnitude of this Hamiltonian peaks around the rotational resonance conditions and has a width depending on the number of rotor periods between the $\pi$ pulses. Its interaction strength can be very significant at the $n=0$ condition, when the chemical shift anisotropies of the interacting spins are of the order of their isotropic chemical shift differences. (C) 2000 American Institute of Physics.

[S0021-9606(00)03203-7]

\section{INTRODUCTION}

Magic angle spinning (MAS) nuclear magnetic resonance (NMR) correlation spectroscopy in conjunction with multispin isotope enrichment has been established recently as a novel concept for refinement of electronic and spatial structure in solids. ${ }^{1,2}$ This development was triggered by the discovery of broadbanded pulse sequences for homonuclear dipolar recoupling. ${ }^{3-11}$ In principle, MAS averages dipolar interactions. ${ }^{12,13}$ Using dipolar recoupling techniques, homonuclear and heteronuclear dipolar correlation spectroscopy can be performed and the NMR response of moderately sized multispin clusters can be assigned. ${ }^{14,15}$ In addition, since the dipolar interaction is strongly distance dependent, dipolar correlation spectroscopy can be used for de novo structure determination on an atomic scale of multispin labeled systems. ${ }^{1,2}$ In this article we present a formal quantized field interpretation of the seminal radio frequency driven dipolar recoupling (RFDR). The technique was originally proposed for one refocusing $\pi$-pulse per single rotor period. ${ }^{5}$ RFDR is straightforward to implement and robust, and can be applied readily in high and ultra high fields. It has already been utilized for assignment and structure refinement of moderately sized uniformly labeled biological aggregates. ${ }^{16}$

It has been shown that the effect of MAS on a nuclear spin system in a solid can be described in terms of a quantized rotor field, leading to a physical interpretation of the

a) Present address: ID-DLO, P.O. Box 65, 8200 AB Lelystad, The Netherlands.
Floquet description of MAS. ${ }^{17,18}$ This makes it possible to disentangle the influence of the radiation field and the MAS rotation, leading to the construction of a Floquet Hamiltonian for the MAS NMR from first principles. Here it will be shown that the quantized field description can be combined with the average Hamiltonian approach in order to provide us with a quasiclassical description of RFDR experiments with a variable number of rotor periods between the $\pi$-pulses. ${ }^{19}$

The formal description of the RFDR experiment starts with a brief summary of the quantum rotor dynamics in the next section. The interaction of the quantized rotor field with the nuclear spin is discussed in Sec. III. The chemical shielding of the magnetic field is calculated in this section and the term in the quantized field Hamiltonian describing the dipolar interaction is put forward. In Sec. IV, the RF pulse is included in the quantized field framework. Using the methodology discussed in Secs. II-IV, we analyze in Sec. V a generalized RFDR sequence. In Sec. VI the polarization transfer driven by the dipolar recoupling sequence is discussed and in Sec. VII the main conclusions are given. In the appendix the transformation properties for the quantized field operators constituting the spin-pair dipolar term in the multispin Hamiltonian are evaluated.

\section{QUANTUM ROTOR DYNAMICS}

MAS NMR spectroscopy is generally described with the help of a spin Hamiltonian, in which the effect of the sample 
rotations on the nuclear spins is taken into account by a set of periodically time dependent coefficients. The spin Hamiltonian of a single crystallite in the rotating frame can therefore be expanded in a Fourier series according to ${ }^{20}$

$$
\begin{aligned}
\hat{\mathcal{H}}_{S}(t)= & \hat{\mathcal{H}}_{S 0}+\sum_{p=1}^{2} \hat{\mathcal{H}}_{S p} \exp \left(i p\left(\varphi_{0}+\omega_{r} t\right)\right)+\hat{\mathcal{H}}_{S p}^{\dagger} \\
& \times \exp \left(-i p\left(\varphi_{0}+\omega_{r} t\right)\right),
\end{aligned}
$$

in which $\omega_{r}$ is the spinning speed and $\hat{\mathcal{H}}_{S p}$ the Fourier components of the Hamiltonian. These components differ from crystallite to crystallite in the sample, while $\varphi_{0}$ is a constant initial phase that may vary between experiments.

In the quantized field description the concept of a nuclear spin Hamiltonian is extended with quantum rotor dynamics. ${ }^{17}$ The interaction of the spins with the environment due to the MAS rotation is included in the Hamiltonian, by using a set of quantum rotor dynamics operators $\hat{L}_{z^{\prime}}, \hat{F}_{p}$, and $\hat{F}_{p}^{\dagger}$, to replace the phenomenological time-dependent phase of the rotation $\varphi_{0}+\omega_{r} t$ in Eq. (1).

Recently, it was shown that rotor field quantization offers a self-consistent framework to describe the evolution of the spin system with conservation of energy. ${ }^{17}$ The total orbital angular momentum $l$ of a forced rotation of a rotor with eigenstates $|l m\rangle$ in the quasiclassical limit defined by $l \rightarrow \infty$ and $l=m$, corresponds with the number of boson quasiparticles. The associated rotor field is a vector field that can be constructed using boson annihilation and creation operators

$$
\begin{aligned}
& \hat{a}|l\rangle=l^{1 / 2}|l-1\rangle, \\
& \hat{a}^{\dagger}|l\rangle=(l+1)^{1 / 2}|l+1\rangle,
\end{aligned}
$$

where

$$
\hat{L}_{z^{\prime}}=\hat{a}^{\dagger} \hat{a}
$$

measures the orbital angular momentum. The energy of the rotor is $\omega_{r} l$, which is very large on the energy scale of the nuclear spin system.

It was shown that a complete description of the MAS for a nuclear magnetic moment in a solid can be obtained within a single set of reduced angular momentum states $|n\rangle$. By taking $l=\bar{n}+n$, with $n$ the variation of the angular momentum around a large average value $\bar{n}$, the orbital angular momentum states can be renumbered according to ${ }^{17}$

$$
|\bar{n}+n\rangle \rightarrow|n\rangle .
$$

To provide a description of the quasiclassical limit, a phase operator $\hat{\varphi}^{\prime}$ is introduced that represents a continuous coordinate $\varphi^{\prime}$ with

$$
\exp \left(i \hat{\varphi}^{\prime}\right)=\frac{\hat{a}^{\dagger}}{\sqrt{\bar{n}}} .
$$

The relation between the phase operator and the angular momentum operator $\hat{L}_{z^{\prime}}$, is the canonical commutation relation according to

$$
\left[\hat{\varphi}^{\prime}, \hat{L}_{z^{\prime}}\right]=i,
$$

and the $z$ axis is the axis of rotation.
MAS is a rotation with a single normal frequency mode $\omega_{r}$. The interaction between the spin system and the sample rotation can be interpreted in terms of $p$-quantum interactions with the single mode rotor field with a frequency $\omega_{r}$. For a $p$-quantum transition, a Fourier operator is defined by

$$
\hat{F}_{p}=\exp \left(i p \hat{\varphi}^{\prime}\right) \text {. }
$$

According to Eq. (5) the $F_{p}$ operators are multiquantum ladder operators with $\hat{F}_{p}|n\rangle=|n+p\rangle$ and they can be interpreted as a repeated application of single quantum raising and lowering operators, since $\hat{F}_{p+q}=\hat{F}_{p} \hat{F}_{q} \cdot{ }^{17}$

The commutator $\left[\hat{L}_{z^{\prime}}, \hat{F}_{p}\right]=p \hat{F}_{p}$ implies that

$$
\begin{aligned}
& \exp \left(i \omega_{r} t \hat{L}_{z^{\prime}}\right) \hat{F}_{p} \exp \left(-i \omega_{r} t \hat{L}_{z^{\prime}}\right) \\
& \quad=\hat{1}+i \omega_{r} t\left[\hat{L}_{z^{\prime}}, \hat{F}_{p}\right]+\frac{\left(i \omega_{r} t\right)^{2}}{2 !}\left[\hat{L}_{z^{\prime}},\left[\hat{L}_{z^{\prime}}, \hat{F}_{p}\right]\right]+\cdots \\
& \quad=\exp \left(i p \omega_{r} t\right) \hat{F}_{p} .
\end{aligned}
$$

In the quasiclassical limit, quantization of the rotor field is therefore equivalent with the introduction of a phase operator $\hat{\varphi}^{\prime}$ in the rotor angle frame according to Eq. (5). A quantized field extension of the spin Hamiltonian in Eq. (1) is then

$$
\begin{aligned}
\hat{\mathcal{H}}_{Q}(t)= & \hat{\mathcal{H}}_{S 0}+\sum_{p=1}^{2} \hat{\mathcal{H}}_{S p} \exp \left(i p\left(\hat{\varphi}^{\prime}+\omega_{r} t\right)\right)+\hat{\mathcal{H}}_{S p}^{\dagger} \\
& \times \exp \left(-i p\left(\hat{\varphi}^{\prime}+\omega_{r} t\right)\right) .
\end{aligned}
$$

It includes two independent degrees of freedom, $\hat{\varphi}^{\prime}$ and $t$, which contrasts with the single time degree of freedom that is used to generate the time-dependence in the spin Hamiltonian $\hat{\mathcal{H}}_{S}(t)$ in Eq. (1). ${ }^{17}$ The $\hat{\varphi}^{\prime}$ degree of freedom is essential, to account for the infinitesimal phase variations due to the quantum rotor dynamics, in which the rotor is described as a two dimensional harmonic oscillator or a rigid rotor. $^{21,22}$

Using the relations (7) and (8), it is possible to rewrite the Eq. (9), yielding

$$
\begin{aligned}
\hat{\mathcal{H}}_{Q}(t)= & \exp \left(i \omega_{r} t \hat{L}_{z^{\prime}}\right)\left(\hat{\mathcal{H}}_{S 0}+\sum_{p=1}^{2} \hat{\mathcal{H}}_{S p} \hat{F}_{p}+\hat{\mathcal{H}}_{S p}^{\dagger} F_{p}^{\dagger}\right) \\
& \times \exp \left(-i \omega_{r} t \hat{L}_{z^{\prime}}\right) .
\end{aligned}
$$

A time-independent Hamiltonian $\hat{\mathcal{H}}_{Q}$ can be obtained by a transformation to the spatial rotating frame, defined by $\exp \left(-i \omega_{r} t \hat{L}_{z^{\prime}}\right)::^{17}$

$$
\hat{\mathcal{H}}_{Q}=\hat{\mathcal{H}}_{n}+\hat{\mathcal{H}}_{i}+\hat{\mathcal{H}}_{r}
$$

consisting of a term associated with the sample rotation

$$
\hat{\mathcal{H}}_{r}=\omega_{r} \hat{L}_{z^{\prime}},
$$

a pure nuclear spin Hamiltonian term independent of $F_{p}$

$$
\hat{\mathcal{H}}_{n}=\hat{\mathcal{H}}_{S 0},
$$

and an interaction term depending on $F_{p}$ and $F_{p}^{\dagger}$

$$
\hat{\mathcal{H}}_{i}=\sum_{p=1}^{2} \hat{\mathcal{H}}_{S p} \hat{F}_{p}+\hat{\mathcal{H}}_{S p}^{\dagger} \hat{F}_{p}^{\dagger},
$$


which describes the effect of the rotor dynamics on the spin interactions. Since $\hat{\mathcal{H}}_{Q}$ is time independent its unitary evolution operator is

$$
\hat{U}(t)=\exp \left(-i \hat{\mathcal{H}}_{Q} t\right) .
$$

The effect of the phenomenological time dependence on the spins is included by means of the time-independent orbital angular momentum ladder operators $\hat{F}_{p}, \hat{F}_{p}^{\dagger}$. These have a physical basis and have been identified as the Fourier operators in the Floquet description of MAS NMR. ${ }^{17}$ The $\hat{\mathcal{H}}_{Q}$ Hamiltonian operates in the product space spanned by the spin states and the (reduced) angular momentum states. The commutation relation between the Fourier operators and the orbital angular momentum operator $\left[\hat{L}_{z^{\prime}}, \hat{F}_{p}\right]=p \hat{F}_{p}$ allows for energy exchange between the rotor and the spin system. In addition, it provides a route to coherent superpositions of states involving the quantum rotor dynamics, since in general $\left[\hat{\mathcal{H}}_{r}, \hat{\mathcal{H}}_{i}\right] \neq 0$.

\section{THE INTERACTION HAMILTONIAN}

The Fourier components of the spin Hamiltonian (1), containing chemical shift and dipolar interactions, can be expressed in terms of contractions between $\hat{\mathbf{A}}_{k}$, the irreducible tensor operators of rank $k$ in real space, and $\hat{\mathbf{T}}_{k}$, the irreducible tensor operators of rank $k$ in spin space. ${ }^{23,24}$ In the spin rotating frame only the part of the interactions that commute with the Zeeman Hamiltonian have to be taken into account. An interaction term of the spin-Hamiltonian in the spin rotating frame can therefore be written as

$$
\hat{\mathcal{H}}_{S}(t)=\sum_{k} A_{k 0}(t) \hat{T}_{k 0} .
$$

The time dependent spatial part can be expanded in a Fourier series according to ${ }^{17}$

$$
A_{k 0}(t)=\sum_{p=-k}^{k} \exp \left(i p\left(\omega_{r} t+\varphi_{0}\right)\right) V_{p 0}^{k},
$$

with

$$
\begin{aligned}
V_{p 0}^{k}= & \sum_{s=-k}^{k} \sum_{r=-k}^{k} A_{k r}^{\prime \prime \prime} D_{r s}^{k}\left(\alpha_{c}, \beta_{c}, \gamma_{c}\right) D_{s p}^{k}\left(\alpha_{r}, \beta_{r}, \gamma_{r}\right) \\
& \times D_{p 0}^{k}\left(0, \beta_{m}, \frac{\pi}{4}\right) .
\end{aligned}
$$

The $A_{k r}^{\prime \prime \prime}$ coefficients are the elements of the spatial tensor describing the coefficients of the interaction in the principal axis system (PAS). In Eq. (18) three sets of Euler angles of the Wigner coefficients $D_{i j}^{k}(\alpha, \beta, \gamma)$ describe the transformation from the PAS to a crystal frame $\left(\alpha_{c}, \beta_{c}, \gamma_{c}\right)$, from this crystal frame to the rotor angle frame $\left(\alpha_{r}, \beta_{r}, \gamma_{r}\right)$, and finally from the rotor angle frame to the laboratory frame $\left(0, \beta_{m}, \pi / 4\right){ }^{23}$

The tensor operators in spin space can be written as

$$
\hat{T}_{k 0}=W_{00}^{k} \hat{I}_{0}
$$

in which $W_{00}^{k}$ are some coefficients and $\hat{I}_{0}$ spin operators commuting with $\hat{I}_{z}$. The quantized field Hamiltonian becomes then

$$
\begin{aligned}
\hat{\mathcal{H}}_{Q}(t)= & Z_{00}^{k} \hat{I}_{0}+\sum_{p=1}^{k} Z_{p 0}^{k} \hat{I}_{0} \exp \left(i p\left(\hat{\varphi}^{\prime}+\omega_{r} t\right)\right. \\
& +Z_{p 0}^{k^{*}} \hat{I}_{0} \exp \left(-i p\left(\hat{\varphi}^{\prime}+\omega_{r} t\right)\right.
\end{aligned}
$$

with

$$
Z_{p 0}^{k}=V_{p 0}^{k} W_{00}^{k}
$$

and the fact that the Hamiltonian is Hermitian requires that $Z_{-p 0}=Z_{p 0}^{*}$. After the transformation to the spatial rotating frame the interaction Hamiltonian in Eq. (14) yields

$$
\mathcal{H}_{i}=\sum_{p=1}^{k}\left(Z_{p 0}^{k} \hat{F}_{p}+Z_{p 0}^{k^{*}} \hat{F}_{p}^{\dagger}\right) \hat{I}_{0}
$$

Here we have used again the Fourier operators to include the quantum rotor dynamics, according to Eq. (7).

For a dipolar coupled spin system of like spins under MAS, the Hamiltonian consists of a chemical shift term and a dipolar term. Thus the nuclear spin Hamiltonian and the interaction Hamiltonian can be written as

$$
\begin{aligned}
& \hat{\mathcal{H}}_{n}=\hat{\mathcal{H}}_{n}^{\mathrm{CS}}+\hat{\mathcal{H}}_{n}^{\mathrm{D}}, \\
& \hat{\mathcal{H}}_{i}=\hat{\mathcal{H}}_{i}^{\mathrm{CS}}+\hat{\mathcal{H}}_{i}^{\mathrm{D}} .
\end{aligned}
$$

With the external magnetic field along the $z$ axis, the spin tensor operators in the truncated isotropic chemical shift $(k=0)$ and chemical shift anisotropy $(\mathrm{CSA})(k=2)$ Hamiltonian in Eq. (16) are for a spin $i^{23}$

$$
\begin{aligned}
& \hat{T}_{00}^{i}=-\frac{1}{\sqrt{3}} \gamma H_{0} \hat{I}_{z}^{i}, \\
& \hat{T}_{20}^{i}=\sqrt{\frac{2}{3}} \gamma H_{0} \hat{I}_{z}^{i} .
\end{aligned}
$$

The spatial isotropic chemical shift tensor is of rank 0 with a single element $A_{00}^{\prime \prime \prime}=\bar{A}^{i}$. The CSA interaction is a real symmetric tensor interaction $\sigma$ of rank 2 and the three elements that characterize this tensor in its principal axis system are for a spin $i$

$$
\begin{aligned}
& A_{20}^{\prime \prime \prime}=\sqrt{\frac{3}{2}} \delta^{i}, \\
& A_{2 \pm 2}^{\prime \prime \prime}=\frac{1}{2} \eta \delta^{i},
\end{aligned}
$$

with $\bar{A}^{i}=\bar{\sigma}^{i}=\sqrt{1 / 3}\left(\sigma_{x x}^{i}+\sigma_{y y}^{i}+\sigma_{z z}^{i}\right)$ the isotropic and $\delta^{i}$ $=1 / 3\left(\sigma_{z z}^{i}-\bar{\sigma}^{i}\right)$ the anisotropy parameter and $\eta^{i}=\left(\sigma_{y y}^{i}\right.$ $\left.-\sigma_{x x}^{i}\right) / \delta^{i}$ the asymmetry parameter of the interaction. ${ }^{25}$ The values of these parameters can be inserted in Eq. (18) to evaluate the $V_{p q}^{k}$ parameters, yielding 


$$
\begin{aligned}
V_{p 0}^{2 i}= & d_{p 0}^{2}\left(\beta_{m}\right) \exp \left(i \gamma_{r} p\right) \sum_{s=-2}^{2}\left(d_{s p}^{2}\left(\beta_{r}\right) \exp \left(i\left(\alpha_{r}+\gamma_{c}^{i}\right) s\right)\right. \\
& \times\left(d_{0 s}^{2}\left(\beta_{c}^{i}\right) \sqrt{\frac{3}{2}} \delta^{i}+\left\{\exp \left(i 2 \alpha_{c}^{i}\right) d_{2 s}^{2}\left(\beta_{c}^{i}\right)\right.\right. \\
& \left.\left.\left.+\exp \left(-i 2 \alpha_{c}^{i}\right) d_{-2 s}^{2}\left(\beta_{c}^{i}\right)\right\} \frac{1}{2} \eta^{i} \delta^{i}\right)\right)
\end{aligned}
$$

with $V_{00}^{2 i}=0$, since for MAS $d_{00}^{2}\left(\beta_{m}\right)=0$.

Using Eqs. (19) and (22), the chemical shift contribution to the interaction Hamiltonian can be constructed and yields

$$
\hat{\mathcal{H}}_{i}^{\mathrm{CS}}=\sum_{i=1}^{N} \sum_{p=1}^{2}\left(Z_{p}^{i} \hat{F}_{p}+Z_{p}^{i^{*}} F_{p}^{\dagger}\right) \hat{I}_{z}^{i},
$$

with $Z_{p}^{i}=\left(Z_{p 0}^{2}\right)^{i}$ and

$$
Z_{p}^{i}=\sqrt{\frac{2}{3}} \gamma H_{0} V_{p 0}^{2 i}
$$

The isotropic part is not affected by the spinning and yields a contribution to the pure nuclear spin term

$$
\hat{\mathcal{H}}_{n}^{\mathrm{CS}}=\sum_{i=1}^{N} \Delta \bar{\omega}^{i} \hat{I}_{z}^{i},
$$

with $\Delta \bar{\omega}^{i}=-\gamma H_{0} 1 / 3\left(\sigma_{x x}^{i}+\sigma_{y y}^{i}+\sigma_{z z}^{i}\right)$.

The spin space tensor operator for the dipolar interaction between a pair of spins $(i, j)$ is

$$
\hat{T}_{20}^{i j}=-\sqrt{\frac{1}{6}} 2 \gamma^{2} \hbar\left(2 \hat{I}_{z}^{i} \hat{I}_{z}^{j}-\frac{1}{2}\left(\hat{I}_{+}^{i} \hat{I}_{-}^{j}+\hat{I}_{+}^{i} \hat{I}_{-}^{j}\right)\right) .
$$

The components of the spatial tensor $V_{p 0}^{2 i j}$ for the spin pair interaction can be obtained from Eq. (26) with $\delta^{i}$ substituted by

$$
\delta^{i j}=-\frac{\mu_{0}}{2 \pi} \frac{\gamma^{2} \hbar}{r_{i j}^{3}},
$$

and $\eta^{i}$ substituted by $\eta^{i j}=0$. Thus the dipolar term in the interaction Hamiltonian is a quantized field contribution that is bilinear in the spin operators,

$\hat{\mathcal{H}}_{i}^{\mathrm{D}}=\sum_{i<j} \sum_{p=1}^{2}\left(Z_{p}^{i j} \hat{F}_{p}+Z_{p}^{i j^{*}} \hat{F}_{p}^{\dagger}\right)\left(2 \hat{I}_{z}^{i} \hat{I}_{z}^{j}-\frac{1}{2}\left(\hat{I}_{+}^{i} \hat{I}_{-}^{j}+\hat{I}_{+}^{i} \hat{I}_{-}^{j}\right)\right)$

with the summation over all spin pairs $i<j$.

Here, with $Z_{p}^{i j}=\left(Z_{p 0}^{2}\right)^{i j}$,

$$
Z_{p}^{i j}=-\frac{C_{p}^{i j}}{r_{i j}^{3}}
$$

and

$$
\begin{aligned}
C_{p}^{i j}= & \frac{\mu_{0}}{4 \pi} \gamma^{2} \hbar d_{p 0}^{2}\left(\beta_{m}\right) \exp \left(i \gamma_{r} p\right) \sum_{s=-2}^{2} d_{s p}^{2}\left(\beta_{r}\right) \\
& \times \exp \left(i\left(\alpha_{r}+\gamma_{c}^{i j}\right) s\right) d_{0 s}^{2}\left(\beta_{c}^{i j}\right)
\end{aligned}
$$

with $\beta_{c}^{i j}$ and $\gamma_{c}^{i j}$ the polar angles of the dipolar vector in the crystal frame.

Since the dipolar interaction is traceless, the contribution to the pure nuclear term due to the dipolar interaction is
$\hat{\mathcal{H}}_{n}^{\mathrm{D}}=0$. According to Eq. (11) the time independent quantized field MAS Hamiltonian $\hat{\mathcal{H}}_{Q}$ of the coupled spin system is the sum of Eqs. (27), (29), (31), and (12).

\section{RADIO FREQUENCY PULSES}

An on-resonance RF field contributes to the spatial rotating frame Hamiltonian a nuclear spin Hamiltonian term of the form:

$$
\hat{\mathcal{H}}_{\mathrm{RF}}=\omega_{1}\left(\hat{I}_{x} \cos \phi+\hat{I}_{y} \sin \phi\right) .
$$

The $\omega_{1}$ is the nutation frequency and $\phi$ is the RF phase in the rotating frame. ${ }^{23}$ The $\hat{\mathcal{H}}_{\mathrm{RF}}$ operates only on the spin part of the spin system and is independent of the sample spinning. If the intensity of a short RF pulse is sufficiently strong then it is possible to neglect all the other terms in the Hamiltonian containing spin operators. During a pulse the effect of the rotor should be considered to account for refocusing errors and to achieve stable effective dipolar recoupling. ${ }^{26}$ The remaining terms in the quantized field Hamiltonian are then

$$
\hat{\mathcal{H}}_{Q}^{\mathrm{RF}}=\hat{\mathcal{H}}_{\mathrm{RF}}+\hat{\mathcal{H}}_{r} .
$$

The $\hat{\mathcal{H}}_{Q}^{\mathrm{RF}}$ is time-independent and its evolution operator at the end of a pulse of length $\tau_{p}$ becomes

$$
\hat{U}\left(\tau_{p}\right)=\exp \left(-i\left(\hat{\mathcal{H}}_{\mathrm{RF}}+\hat{\mathcal{H}}_{r}\right) \tau_{p}\right) .
$$

However, in the limit of an extremely short pulse $\tau_{p} \rightarrow 0$, while $\theta=\omega_{1} \tau_{p}$ is kept constant, also the rotor dynamics during the pulse can be neglected and a pulse evolution operator can be defined as

$$
\hat{P}(\theta)=\exp \left(-i \sum_{i=1}^{N} \theta\left(\hat{I}_{x}^{i} \cos \phi+\hat{I}_{y}^{i} \sin \phi\right)\right),
$$

affecting only the spin-operational part of the spin system. $^{27,28}$

During an interval of length $\tau_{m}$ between such pulses, $\hat{P}_{m-1}$ and $\hat{P}_{m}$, in a multi-pulse experiment the spin system is governed by the time independent quantized field Hamiltonian $\hat{\mathcal{H}}_{Q}$ and the evolution of the spin system in this period is described by the evolution operator $\hat{U}\left(\tau_{m}\right)=\exp$ $\left(-i \hat{\mathcal{H}}_{Q} \tau_{m}\right)$. The overall evolution operator for a pulse sequence of $M$ equally spaced pulses during a time of $\tau_{c}$ is

$$
\hat{\bar{U}}\left(\tau_{c}\right)=\prod_{m=1}^{M} \hat{P}_{m} \hat{U}\left(\tau_{m}\right)
$$

with

$$
\tau_{c} \equiv \sum_{m=1}^{M} \tau_{m}
$$

Schematic representations of such pulse sequences are shown in Fig. 1.

A product of unitary transformations is again a unitary transformation and it should be possible to express the entire sequence in terms of an average quantized field Hamiltonian $\widehat{\overline{\mathcal{H}}}_{Q}$ according to

$$
\hat{\bar{U}}\left(\tau_{c}\right)=\exp \left(-i \hat{\overline{\mathcal{H}}}_{Q} \tau_{c}\right),
$$


A
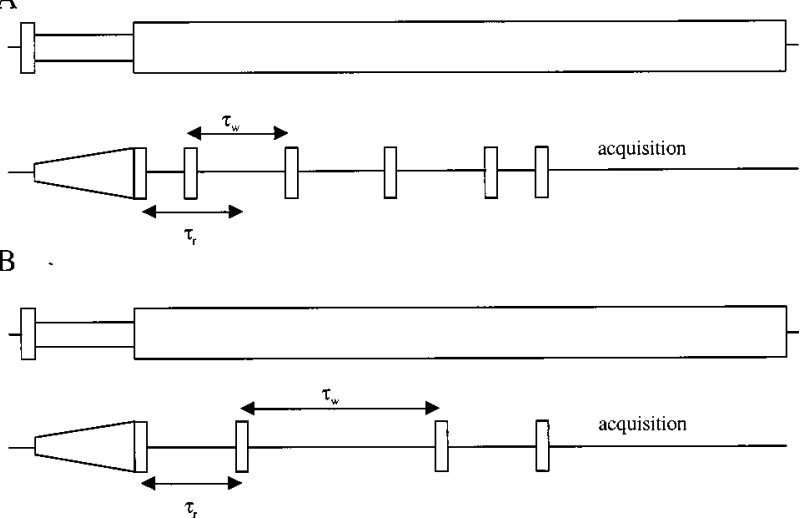

FIG. 1. Schematic representation of the generalized RFDR sequence with (A) $\tau_{w}=\tau_{r}$ and (B) $\tau_{w}=2 \tau_{r}$. The sequences start with ramped cross polarization. The magnetization is rotated to the $z$ axis and the recoupling sequence is applied. Prior to detection the polarization is transferred to the $x y$ plane.

as for spin-Hamiltonians. ${ }^{29}$ The evaluation of this average Hamiltonian must be accomplished by using average Hamiltonian theory in the quantized field representation. This is discussed in the following section.

\section{ROTOR FREQUENCY-DRIVEN DIPOLAR RECOUPLING}

The RFDR sequence was analyzed previously using average Hamiltonian theory and Floquet theory. ${ }^{5,30,31}$ It utilizes a train of rotor-synchronized $\pi$-pulses to invoke the dipolar recoupling. In the original version one pulse per rotor period is used, with a time window between the pulses $\tau_{w}=\tau_{r}$, with $\tau_{r}$ the length of one rotor period. Here a generalization of RFDR is discussed that was first introduced in Ref. 19. A fixed number of rotor periods per pulse $n_{w}$ is used and $\tau_{w}$ $=n_{w} \tau_{r}$ is the time window between the pulses. ${ }^{19,26}$ In this paper we restrict ourselves to even $n_{w}$ values and one RFDR cycle contains two pulses $\left[\frac{1}{2} \tau_{w}-\hat{P}_{1}(\pi)-\tau_{w}-\hat{P}_{2}(\pi)\right.$ $\left.-\frac{1}{2} \tau_{w}\right]$ in a cycle time of $\tau_{c}=2 \tau_{w}$. The sequence allows for narrow band RFDR and its frequency selectivity has been discussed recently in Ref. 26. When the pulses are cyclic in the sense $\Pi_{m=1}^{p} \hat{P}_{1} \hat{P}_{2}=\hat{1}$, the expression for the averaged quantized field Hamiltonian can be found by transforming $\hat{\mathcal{H}}_{Q}$ to the "toggling frame," were the RF pulses are eliminated. ${ }^{27,28}$ This implies that the evolution operator in the spatial rotating frame and the toggling frame are the same at the end of each RFDR cycle.

The basis RFDR pulse cycle can be divided into three periods $0 \leqslant t \leqslant \frac{1}{2} \tau_{w}, \frac{1}{2} \tau_{w} \leqslant t \leqslant \frac{3}{2} \tau_{w}$, and $\frac{3}{2} \tau_{w} \leqslant t \leqslant 2 \tau_{w}$. The effect of such a cycle on an ensemble of $N$ dipolar coupled like spins can be evaluated using the quantized field formalism in the toggling interaction frame of the chemical shift and the sample spinning. If the $\hat{\mathcal{H}}_{Q}$ is divided according to $\hat{\mathcal{H}}_{Q}=\hat{\mathcal{H}}_{0}+\hat{\mathcal{H}}_{1}$, with

$$
\begin{aligned}
& \hat{\mathcal{H}}_{0}=\hat{\mathcal{H}}_{n}^{\mathrm{CS}}+\hat{\mathcal{H}}_{i}^{\mathrm{CS}}+\hat{\mathcal{H}}_{r}, \\
& \hat{\mathcal{H}}_{1}=\hat{\mathcal{H}}_{i}^{\mathrm{D}},
\end{aligned}
$$

the toggling frame Hamiltonian becomes

$$
\begin{aligned}
& \hat{\tilde{\mathcal{H}}}_{0}=\left\{\begin{array}{cc}
\hat{\mathcal{H}}_{n}^{\mathrm{CS}}+\hat{\mathcal{H}}_{i}^{\mathrm{CS}}+\hat{\mathcal{H}}_{r}, & 0<t<\frac{1}{2} \tau_{w}, \\
-\hat{\mathcal{H}}_{n}^{\mathrm{CS}}-\hat{\mathcal{H}}_{i}^{\mathrm{CS}}+\hat{\mathcal{H}}_{r}, & \frac{1}{2} \tau_{w}<t<\frac{3}{2} \tau_{w}, \\
\hat{\mathcal{H}}_{n}^{\mathrm{CS}}+\hat{\mathcal{H}}_{i}^{\mathrm{CS}}+\hat{\mathcal{H}}_{r}, & \frac{3}{2} \tau_{w}<t<2 \tau_{w},
\end{array}\right. \\
& \widehat{\widetilde{\mathcal{H}}}_{1}=\hat{\mathcal{H}}_{i}^{\mathrm{D}}
\end{aligned}
$$

where we assumed infinitely short $\pi$-pulses.

The evolution operator can be written in the toggling frame as $\widehat{\tilde{U}}(t)=\widehat{\tilde{U}}_{0}(t) \widehat{\tilde{U}}_{1}(t)$, with $\widehat{\tilde{U}}_{1}(t)$ governed by the equation of motion

$$
\frac{d \widehat{\widetilde{U}}_{1}(t)}{d t}+i \widehat{\widetilde{\mathcal{H}}}_{\mathrm{int}}(t) \widehat{\tilde{U}}_{1}(t)=0,
$$

with

$$
U_{0}(t)=\exp \left(-i \widehat{\mathcal{H}}_{0} t\right)
$$

and

$$
\widehat{\mathcal{H}}_{\text {int }}(t)=\widehat{\widetilde{U}}_{0}^{-1}(t) \widehat{\mathcal{H}}_{1} \widehat{\widehat{U}}_{0}(t),
$$

the Hamiltonian in the interaction frame of $\widehat{\mathcal{H}}_{0} \cdot{ }^{25}$

The evolution operator $\widetilde{\tilde{U}}_{1}(t)$ in this interaction frame at $t=2 \tau_{w}$ becomes equal to the toggling frame $\widehat{\hat{U}}\left(2 \tau_{w}\right)$ when $\widehat{\widetilde{U}}_{0}\left(2 \tau_{w}\right)=\hat{1}$. This equality can easily be shown by the following calculation and implies that the spin evolution in the original spatial rotating frame is governed by $\hat{U}\left(2 \tau_{w}\right)$ $=\widehat{\widetilde{U}}_{1}\left(2 \tau_{w}\right)$. The Hamiltonian $\widehat{\widetilde{\mathcal{H}}}_{0}$ in Eq. (42) contains three terms. The isotropic chemical shift term, proportional to $\mathcal{H}_{n}^{\text {CS }}$ in Eq. (29), the CSA interaction terms, equal to $\pm \hat{\mathcal{H}}_{i}^{\mathrm{CS}} \mathrm{de}-$ fined in Eq. (27), and the rotor term $\mathcal{H}_{r}$. The first term commutes with the additional terms and its evolution operator is one at $2 \tau_{w}$. Thus the evolution operator in Eq. (44) only depends on the two remaining and noncommuting terms:

$$
\begin{aligned}
\hat{\tilde{U}}_{0}\left(2 \tau_{w}\right)= & \exp \left(-i / 2\left(\hat{\mathcal{H}}_{i}^{\mathrm{CS}}+\hat{\mathcal{H}}_{r}\right) \tau_{w}\right) \exp \left(-i\left(-\hat{\mathcal{H}}_{i}^{\mathrm{CS}}\right.\right. \\
& \left.\left.+\hat{\mathcal{H}}_{r}\right) \tau_{w}\right) \exp \left(-i / 2\left(\hat{\mathcal{H}}_{i}^{\mathrm{CS}}+\hat{\mathcal{H}}_{r}\right) \tau_{w}\right) .
\end{aligned}
$$

To show that this operator equals one we use the following diagonalization conditions:

$$
\begin{aligned}
& \hat{\mathcal{H}}_{i}^{\mathrm{CS}}+\hat{\mathcal{H}}_{r}=\hat{D}^{-1} \hat{\mathcal{H}}_{r} \hat{D}, \\
& -\hat{\mathcal{H}}_{i}^{\mathrm{CS}}+\hat{\mathcal{H}}_{r}=\hat{D} \hat{\mathcal{H}}_{r} \hat{D}^{-1}
\end{aligned}
$$

which are derived in Appendix A. Insertion of these equations into Eq. (46) and using that $\exp \left(i \hat{\mathcal{H}}_{r} \tau_{w}\right)=\hat{1}$ results in a periodic $\widehat{\widetilde{U}}_{0}(t)$ operator with

$$
\begin{aligned}
\widehat{\widetilde{U}}_{0}\left(2 \tau_{w}\right)= & \hat{D} \exp \left(-i / 2 \hat{\mathcal{H}}_{r} \tau_{w}\right) \hat{D}^{-1} \hat{D}^{-1} \\
& \times \exp \left(-i \hat{\mathcal{H}}_{r} \tau_{w}\right) \hat{D} \hat{D} \exp \left(-i / 2 \hat{\mathcal{H}}_{r} \tau_{w}\right) \hat{D}^{-1}=\hat{1}
\end{aligned}
$$

Thus the CSA interaction is refocused at the end of a RFDR cycle, as is well known for two $\pi$-pulses that are separated by an integer multiple of rotor periods $\tau_{w}=n_{w} \tau_{c}$. 
It is now possible to use the interaction Hamiltonian in Eq. (45) for the evaluation of the average RFDR Hamiltonian. The quantized interaction Hamiltonian is time dependent and is not anymore defined in the spatial rotating frame. This is due to the fact that the transformation operator $\overrightarrow{\widetilde{U}}_{0}(t)$ contains the rotor Hamiltonian term $\hat{\mathcal{H}}_{r}$. However, because we are only interested in the spin response at the end of the RFDR cycles and $\widehat{\tilde{U}}_{0}(t)$ is periodic, the time independent average Hamiltonian can be considered as being defined in the spatial rotating frame. We will restrict ourselves to the calculation of the zero order average Hamiltonian assuming that $2 \tau_{w}\left\|\widehat{\mathcal{H}}_{\text {int }}\right\| \ll 1$.

\section{THE ZERO ORDER AVERAGE RFDR HAMILTONIAN}

The zero order average RFDR Hamiltonian of a homonuclear spin pair $(i, j)$, evolving with the isotropic and anisotropic chemical shift interactions of both spins defined in Eqs. (27) and (29), equals

$$
\widehat{\widehat{\mathcal{H}}}_{Q}=\frac{1}{2 \tau_{w}} \int_{0}^{2 \tau_{w}} \widehat{\mathcal{H}}_{\mathrm{int}} d t=\frac{1}{2 \tau_{w}} \int_{0}^{2 \tau_{w}} \widehat{\widetilde{U}}_{0}^{-1}(t) \widehat{\mathcal{\mathcal { H }}}_{1} \widehat{\widetilde{U}}_{0}(t) d t
$$

with the two-spin dipolar interaction Hamiltonian in Eq. (31). Simplifying the expressions by defining the operators,

$$
\begin{aligned}
& \hat{U}_{r}(t)=\exp \left(-i \hat{\mathcal{H}}_{r} t\right), \\
& \hat{U}_{n}^{\mathrm{CS}}(t)=\exp \left(-i \sum_{i=1}^{2} \Delta \bar{\omega}^{i} \hat{I}_{z}^{i} t\right)
\end{aligned}
$$

and using the diagonalization operators $\hat{D}=\hat{D}_{i} \hat{D}_{j}$ of the two spins, the integral of the average Hamiltonian can be written as

$$
\begin{aligned}
\widehat{\mathcal{\mathcal { H }}}_{Q}= & \frac{1}{2 \tau_{w}} \int_{-1 / 2 \tau_{w}}^{1 / 2 \tau_{w}} d t \hat{U}_{n}^{\mathrm{CS}^{-1}}(t) \\
& \times\left\{\hat{D} \hat{U}_{r}(t) \hat{D}^{-1}\right\} \hat{\mathcal{H}}_{1}\left\{\hat{D} \hat{U}_{r}^{-1}(t) \hat{D}^{-1}\right\} \hat{U}_{n}^{\mathrm{CS}}(t) \\
& +\hat{U}_{n}^{\mathrm{CS}}(t)\left\{\hat{D}^{-1} \hat{U}_{r}(t) \hat{D}\right\} \hat{\mathcal{H}}_{1} \\
& \times\left\{D^{-1} \hat{U}_{r}^{-1}(t) \hat{D}\right\} U_{0}^{-1}(t) .
\end{aligned}
$$

Here we used the fact that during the three time intervals of the RDFR cycle the Hamiltonians are time independent. In the appendix the integral in Eq. (51) is calculated by expanding the diagonalization operator. The result yields an expression for $\widehat{\overline{\mathcal{H}}}_{Q}$ of the form:

$$
\begin{aligned}
\widehat{\mathcal{H}}_{Q} & =-\sum_{i<j}\left(d_{i j}^{0}+\sum_{k=1}^{\infty} d_{i j}^{k} \hat{F}_{k}+d_{i j}^{k^{*}} \hat{F}_{k}^{\dagger}\right) \frac{1}{2}\left(\hat{I}_{+}^{i} \hat{I}_{-}^{j}+\hat{I}_{-}^{i} \hat{I}_{+}^{j}\right) \\
& =-\sum_{i<j}\left(d_{i j}^{0}+\sum_{k=1}^{\infty} d_{i j}^{k} \hat{F}_{k}+d_{i j}^{k^{*}} \hat{F}_{k}^{\dagger}\right)\left(\hat{I}_{x}^{i} \hat{I}_{x}^{j}+\hat{I}_{y}^{i} \hat{I}_{y}^{j}\right),
\end{aligned}
$$

with

$$
d_{i j}^{k}=\frac{1}{r_{i j}^{3}} \sum_{n=-\infty}^{\infty} M_{k n}^{i j} \operatorname{sinc}\left(\frac{2 n_{w}\left(\Delta \bar{\omega}^{i}-\Delta \bar{\omega}^{j}-n \omega_{r}\right)}{\omega_{r}}\right)
$$

and

$$
M_{k n}^{i j}=-\sum_{p=-2}^{2} C_{p}^{i j}\left(K_{n+k}^{i j} K_{n+p}^{i j *}+K_{n-k}^{i j *} K_{n-p}^{i j}\right)
$$

with

$$
\begin{aligned}
K_{m}^{i j}= & \sum_{k=-\infty}^{\infty} J_{k}\left(-\frac{\left|Z_{2}^{i}-Z_{2}^{j}\right|}{\omega_{r}}\right) J_{m-2 k}\left(-2 \frac{\left|Z_{1}^{i}-Z_{1}^{j}\right|}{\omega_{r}}\right) \\
& \times \exp \left(i(m-2 k) \phi_{1}^{i j}+i k \phi_{2}^{i j}\right),
\end{aligned}
$$

where $C_{p}^{i j}$ is given by Eq. (33) and $Z_{p}^{i j}$ in Eq. (32). In Eq. (52) a summation over all spin pairs in a coupled homonuclear spin system is added. The $\hat{\overrightarrow{\mathcal{H}}}_{Q}$ can induce polarization transfer between the nuclei. ${ }^{5}$ The rate of transfer strongly depends on the internuclear distance $d_{i j}^{k} \propto 1 / r_{i j}^{3}$, according to Eq. (53). This provides the selectivity necessary for correlation spectroscopy in isotope enriched solids.

Introducing the deviation from the rotational resonance condition by the off-rotational resonance value $\delta \bar{\omega}_{i j}^{n}=\left(\Delta \bar{\omega}^{i}\right.$ $-\Delta \bar{\omega}^{j}-n \omega_{r}$ ), we see that the sinc-function in Eq. (53) has a maximum at every rotational resonance condition $\delta \bar{\omega}_{i j}^{n}=0$, including the $n=0$ rotational resonance condition. For $\delta \bar{\omega}_{i j}^{n}$ $\neq 0$ the sinc-function decreases and can become zero for a certain value. If we define the bandwidth of the RFDR as the difference between the two zero points around the maximum value, the bandwidth is ${ }^{19}$

$$
B_{w}=\frac{\omega_{r}}{n_{w}} .
$$

This implies that the bandwidth can be narrowed by increasing the number of rotor periods per pulse. In the limit of large windows between the pulses $\left(n_{w} \rightarrow \infty\right)$ this bandwidth goes to zero. In this case only at the rotational resonance condition the zero order average Hamiltonian recovers the dipolar interaction. With adding $\pi$-pulses according to the extended RFDR scheme, the rotational resonance condition becomes broader and spectral selectivity can be considered. In this way, the rotor frequency drives the transfer in RFDR and not the radio frequency.

In Eq. (53) also the $n=0$ rotational resonance is included. This implies that also for small isotropic shift differences the dipolar interaction can be recovered. In the early treatment of the RFDR, this possibility for efficient transfer with small isotropic shift differences has been overlooked, since the shift anisotropy was generally excluded from the earlier analytical treatments. In contrast, in our quantized field analysis the chemical shift anisotropy is included and is embedded in the $K_{m}^{i j}$ in Eq. (55). This theoretical outcome is experimentally confirmed, because efficient transfer around $n=0$ has been observed in chlorophylls. ${ }^{14,16}$ It can be concluded that RFDR is considerably more broadband than originally thought.

\section{POLARIZATION TRANSFER}

In Eq. (52) the quantized field average Hamiltonian is given for the RFDR cycle. This Hamiltonian can be used to describe the evolution of the spin system after $n_{m}$ cycles via the evolution operator 


$$
\hat{\bar{U}}\left(\tau_{m}\right)=\left[\exp \left(-i 2 \hat{\overline{\mathcal{H}}}_{Q} \tau_{w}\right)\right]^{n_{m}}=\exp \left(-i \hat{\overline{\mathcal{H}}}_{Q} \tau_{m}\right),
$$

with $\tau_{m}=2 n_{m} \tau_{w}$. This propagator promotes polarization transfer from nucleus $i$ to nucleus $j$. Within the multi-spin formalism presented here, multiple pathways are implicitly covered, since the polarization transfer can be direct

$$
\begin{gathered}
\hat{\overline{\mathcal{H}}}_{Q} \\
i \rightarrow j,
\end{gathered}
$$

or can be relayed, e.g.,

$$
\begin{gathered}
\hat{\overline{\mathcal{H}}}_{Q} \hat{\overline{\mathcal{H}}}_{Q} \\
i \rightarrow k \rightarrow j .
\end{gathered}
$$

The discrimination between direct and relayed transfer has attracted considerable attention in recent years. ${ }^{32}$ In a general treatment, the evolution of the spin system and the transfer of polarization can be described in terms of the evolution of the density operator

$$
\hat{\rho}\left(\tau_{m}\right)=\hat{\hat{U}}\left(\tau_{m}\right) \hat{\rho}(0) \hat{\bar{U}}^{-1}\left(\tau_{m}\right) .
$$

The density operator in the quantized field representation describes the combination of rotor and spin system. This approach is very demanding, because the total process of creation and annihilation of particles via the Fourier operators in combination with the multiple spin processes has to be taken into account. Therefore, to simplify matters we project the quantized Hamiltonian onto an average spin Hamiltonian $\hat{\mathcal{H}}_{S}$ via a "dequantization" of the phase: $\hat{F}_{k}$ $=\exp \left(i k \hat{\varphi}^{\prime}\right) \rightarrow \exp \left(i k \varphi_{0}\right)$. This is justified in the simple case we intend to consider, provided we are in the limit of a large number of rotor quanta. The average Hamiltonian in Eq. (52) becomes then

$$
\hat{\overline{\mathcal{H}}}_{S}=-\sum_{i<j} \bar{d}_{i j}\left(\hat{I}_{x}^{i} \hat{I}_{x}^{j}+\hat{I}_{y}^{i} \hat{I}_{y}^{j}\right)
$$

with

$$
\bar{d}_{i j}=\left(d_{i j}^{0}+\sum_{k=1}^{\infty} d_{i j}^{k} \exp \left(i k \varphi_{0}\right)+d_{i j}^{k^{*}} \exp \left(-i k \varphi_{0}\right)\right) .
$$

If we restrict ourselves to a two spin system then we avoid relayed polarization transfer. A convenient way of describing a two-spin system is using the single-transition operators ${ }^{33,34}$

$$
\begin{aligned}
& I_{z}^{23}=\frac{1}{2}\left(\hat{I}_{z}^{1}-\hat{I}_{z}^{2}\right), \\
& I_{x}^{23}=\left(\hat{I}_{x}^{1} \hat{I}_{x}^{2}+\hat{I}_{y}^{1} \hat{I}_{y}^{2}\right), \\
& I_{y}^{23}=\left(\hat{I}_{y}^{1} \hat{I}_{x}^{2}-\hat{I}_{x}^{1} \hat{I}_{y}^{2}\right) .
\end{aligned}
$$

The average Hamiltonian in Eq. (59) can be expressed in those operators, according to

$$
\hat{\mathcal{H}}_{S}=-\sum_{i<j} \bar{d}_{i j} \hat{I}_{x}^{23}
$$

If the spin density operator at the beginning of the RFDR period is

$$
\hat{\rho}(0)=\left(\hat{I}_{z}^{1}-\hat{I}_{z}^{2}\right)=2 I_{z}^{23},
$$

the average Hamiltonian will lead to rotation in the $z y$ plane around the $x$ axis of this space of single-transition operators, according to

$$
\hat{\rho}\left(\tau_{m}\right)=\left(2 \hat{I}_{z}^{23} \cos \left(\bar{d}_{12} \tau_{m}\right)+2 \hat{I}_{y}^{23} \sin \left(\bar{d}_{12} \tau_{m}\right)\right.
$$

which is consistent with earlier findings. ${ }^{5,31}$ However, in $\bar{d}_{i j}$ also the CSA and the narrow band RFDR sequence are taken into account. This implies that by measuring $\bar{d}_{i j}$ as a function of the $n_{w}$ in principle not only information can be deduced about the internuclear distance, but also about the relative orientation of the CSA tensors of the two sites. ${ }^{35}$

This is illustrated in Fig. 2 with simulations of a RFDR experiment on two ${ }^{13} \mathrm{C}$ spins resonating with a Larmor frequency of $125.7 \mathrm{MHz}$ and separated by $\sim 1.4 \AA$ with a small isotropic shift difference of $14 \mathrm{ppm}$, corresponding with 1.76 $\mathrm{kHz}$. The orientation of the PAS of the CSA for the second spin is defined with respect to the PAS of the CSA of the first spin and changed by varying the Euler angle $0 \leqslant \beta \leqslant 180$. For $\beta=0$ the two tensors are aligned and the dipolar vector is along the axis corresponding with the largest principle component of the spin 1. The points are evaluated at three different spinning speeds $\nu_{r}=\omega_{r} / 2 \pi$ assuming an initial condition for the density matrix $\left\langle 2 \hat{I}_{z}^{1}\right\rangle$ and calculating the $\left\langle 2 \hat{I}_{z}^{2}\right\rangle$ after a constant mixing time $\tau_{m}=2.66 \mathrm{~ms}$.

At a low speed $\nu_{r}=6 \mathrm{kHz}$ we calculate efficient transfer for $n_{w}=1$ with $\left\langle 2 \hat{I}_{z}^{2}\right\rangle$ approaching the maximum of $\sim 0.5$ over a broad range and it depends on the value of $\beta$. The transfer efficiency drops for $n_{w}=2$ and the angular dependence is enhanced. The narrow band character of the RFDR is nicely illustrated for $n_{w}=4$. Here the bandwidth is $B_{w} / 2 \pi=1.5 \mathrm{kHz}$, less than the isotropic shift difference $\left(\Delta \bar{\omega}^{1}-\Delta \bar{\omega}^{2}\right) / 2 \pi=1.76 \mathrm{kHz}$ and the $\left\langle 2 \hat{I}_{z}^{2}\right\rangle \sim 0.1$, much less than for the $n_{w}=1$ and $n_{w}=2$ at the same spinning speed. This reflects the decrease of the bandwidth for increasing $n_{w}$.

With a spinning speed of $12 \mathrm{kHz}$, we calculate again a considerable dependence of the transfer efficiency on the angle $\beta$, which is most pronounced around $\beta=30^{\circ}$ and $\beta=150^{\circ}$. The $\left\langle 2 \hat{I}_{z}^{2}\right\rangle$ goes through a maximum around $\beta=90^{\circ}$. The relative variation of the transfer with $\beta$ is strongest for $n_{w}=4$. The $\left\langle 2 \hat{I}_{z}^{2}\right\rangle$ peaks around $\beta=90^{\circ}$ and it almost vanishes for $\beta=0^{\circ}$ or $\beta=180^{\circ}$. These calculations suggest new and interesting possibilities for collecting angular information by measuring the transfer rate for different $n_{w}$, using the generalized RFDR treatment discussed here for the analysis. At $24 \mathrm{kHz}$, the transfer characteristics change again. At the high speed of $24 \mathrm{kHz}$ also for the $n_{w}=4$ the $B_{w} / 2 \pi=6 \mathrm{kHz}$ is sufficient to cover the isotropic shift difference of $1.76 \mathrm{kHz}$. In addition, the spinning speed of 24 $\mathrm{kHz}$ is sufficient to attenuate the CSA's, since $\delta^{1} / 2 \pi$ $=-11.3 \mathrm{kHz}$ and $\delta^{2} / 2 \pi=-11.7 \mathrm{kHz}$. The $M_{k n}^{i j}$ in Eq. (54) depend on the spinning speed. They vanish in the limit of $\omega_{r} \rightarrow \infty$. Therefore the transfer is less efficient at high speed, with $\left\langle 2 \hat{I}_{z}^{2}\right\rangle$ below 0.2 for $\mathrm{v}_{r}=24 \mathrm{kHz}$. This illustrates that close to the $n=0$ rotational resonance condition the presence of a dipolar interaction alone is insuffient for polarization transfer and that there is a prominent effect of the CSA on the transfer efficiency via the $M_{k n}^{i j}$. 


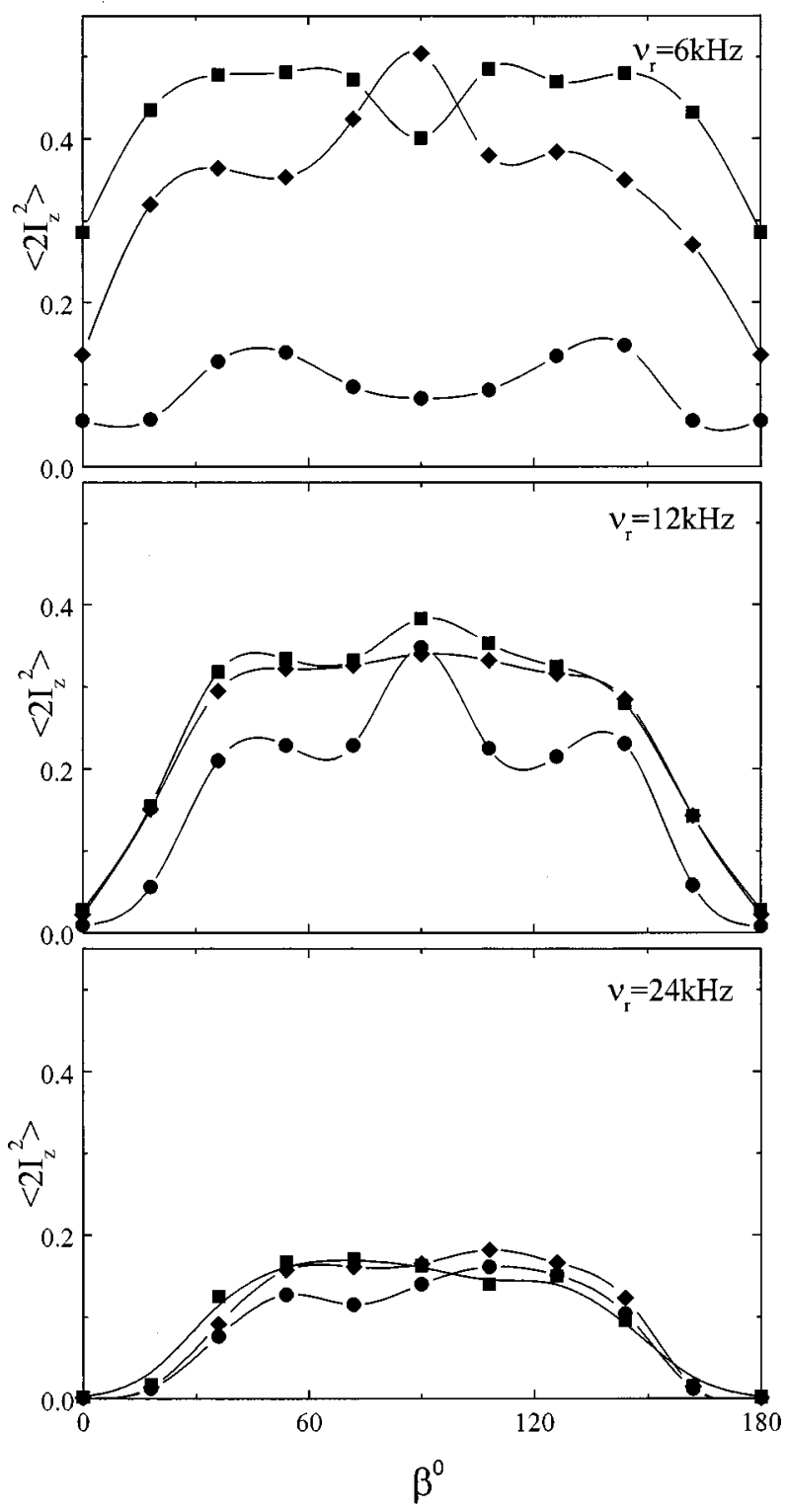

FIG. 2. Examples of magnetization transfer within a dipolar coupled spin pair. The calculations are performed for two spins separated by $14 \mathrm{ppm}$ and resonating at $125.7 \mathrm{MHz}$. A set of parameters was used that correspond to the CSA's that can be observed, e.g., aromatic or ethylenic carbons: for the first spin an anisotropy $\delta^{1}=-90 \mathrm{ppm}$ was used with an asymmetry parameter $\eta=0.41$, while $\delta^{2}=-93 \mathrm{ppm}$ with $\eta=0.73$. In the evaluation of the signals $\varphi_{0}=0$ and 300 orientations are taken for each point. The relative orientation of the tensors is defined by the Euler angles for the second spin $\left(\alpha_{c}^{2}, \beta_{c}^{2}, \gamma_{c}^{2}\right)$, following Eq. (26). In this calculation $\alpha_{c}^{2}=\gamma_{c}^{2}=0$ and $\beta_{c}^{2}$ $=\beta_{0}$ is varied. The Euler angles for the transformation of the PAS of the first spin and for the transformation of the dipolar frame to the crystal frame according to Eq. (33) are taken zero. This corresponds with an orientation of the dipolar vector parallel to the most shielded direction of the first spin $I_{z}^{1}$. The powder integration is performed over $\left(\alpha_{r}, \beta_{r}, \gamma_{r}\right)$. The points are evaluated after $\tau_{\text {mix }}=2.66 \mathrm{msec}$ by assuming an initial condition for the density matrix $2 I_{z}^{1}$ and following the magnitude of $\left\langle 2 I_{z}^{2}\right\rangle$. The maximum value we can expect is $\left\langle 2 I_{z}^{2}\right\rangle \sim 0.5$ in a powder. $X Y 8$ phase cycling was used for the full RFDR ( $n_{w}=1$, squares), $X Y 4$ for $n_{w}=2$ (diamonds), and $X \bar{X}$ for $n_{w}=4$ (circles).

\section{CONCLUDING REMARKS}

We have shown that the quantized field description can be used to analyze pulse sequences including MAS on a multiple spin system. It is demonstrated that the quantized field approach, from which the Floquet approach can be derived, can be combined with average Hamiltonian theory. In the earlier Floquet treatment of the RFDR, an effective Hamiltonian was formulated, with the pulses included in the sequence. ${ }^{30}$ In the quantized field formalism the effect of the MAS rotation and the rotation itself are disentangled and the resulting time-independent Hamiltonian can be treated in an average Hamiltonian formalism by adding the pulses afterwards. In this way average Hamiltonian theory and Floquet theory are fully compatible, since average Hamiltonian theory can be applied to the time dependent quantized field Hamiltonian in the toggling frame.

The $\hat{\overline{\mathcal{H}}}_{Q}$ in Eq. (52) induces polarization transfer between nuclei in a multiple spin system. ${ }^{5}$ The probability of transfer strongly depends on the internuclear distance $\delta_{i j}^{k}$ $\propto 1 / r^{3}$ and this makes correlation spectroscopy in isotope enriched solids possible. The probability of polarization transfer has a maximum at every rotational resonance condition $\bar{\sigma}^{i}-\bar{\sigma}^{j}=n \omega_{r}$. Since the CSA is embedded in the $K_{m}^{i j}$ also the $n=0$ rotational resonance condition is included.

According to our treatment, the driving force in both RFDR and rotational resonance is the rotor frequency and not the radio frequency. This suggests that a physically more appropriate expansion of the RFDR abbreviation would be "rotor frequency-driven dipolar recoupling." The RFDR bandwidth can be changed by changing the window between the $\pi$-pulses. This additional variable can be used to obtain information about relative orientation of the CSA tensors.

\section{ACKNOWLEDGMENTS}

This research was supported by the foundation of Life sciences (ALW), financed by the Netherlands Organization for Scientific Research (NWO) and part of this research was supported by The Israel Science Foundation. G.J.B. and H.J.M.dG are recipients of NWO TALENT and PIONIER awards, respectively.

\section{APPENDIX}

In this appendix the expression for the zero order average Hamiltonian in Eq. (52) is derived. To do so the interaction Hamiltonian of a two-spin system must be calculated by applying the transformation of Eq. (45):

$$
\widehat{\widetilde{\mathcal{H}}}_{\text {int }}(t)=\widehat{\tilde{U}}_{0}^{-1}(t) \widehat{\widetilde{\mathcal{H}}}_{1} \widehat{\tilde{U}}_{0}(t) .
$$

The transformation operator is defined by the sum of the isotropic chemical shift Hamiltonians

$$
\begin{aligned}
& \hat{\widetilde{\mathcal{H}}}_{n}^{\mathrm{CS}}(t) \\
& \quad= \begin{cases}1 / 2\left(\Delta \bar{\omega}^{i}-\Delta \bar{\omega}^{j}\right)\left(I_{z}^{i}-I_{z}^{j}\right), & 0<t<1 / 2 \tau_{w}, \\
-1 / 2\left(\Delta \bar{\omega}^{i}-\Delta^{j}\right)\left(I_{z}^{i}-I_{z}^{j}\right), & 1 / 2 \tau_{w}<t<3 / 2 \tau_{w}, \\
1 / 2\left(\Delta \bar{\omega}^{i}-\Delta \bar{\omega}^{j}\right)\left(I_{z}^{i}-I_{z}^{j}\right), & 3 / 2 \tau_{w}<t<2 \tau_{w},\end{cases}
\end{aligned}
$$

and the CSA interaction term plus the rotor Hamiltonian 


$$
\begin{aligned}
& \widehat{\mathcal{H}}_{i}^{\mathrm{CS}}(t)+\hat{\mathcal{H}}_{r} \\
& =\left\{\begin{array}{l}
\sum_{k=i, j} \sum_{p=1}^{2}\left(Z_{p}^{k} \hat{F}_{p}+Z_{p}^{k^{*}} \hat{F}_{p}^{\dagger}\right) I_{z}^{k}+\hat{\mathcal{H}}_{r}, \quad 0<t<1 / 2 \tau_{w}, \\
-\sum_{k=i, j} \sum_{p=1}^{2}\left(Z_{p}^{k} \hat{F}_{p}+Z_{p}^{k^{*}} \hat{F}_{p}^{\dagger}\right) I_{z}^{i}+\hat{\mathcal{H}}_{r}, \\
1 / 2 \tau_{w}<t<3 / 2 \tau_{w}, \\
\sum_{k=i, j} \sum_{p=1}^{2}\left(Z_{p}^{k} \hat{F}_{p}+Z_{p}^{k^{*}} \hat{F}_{p}^{\dagger}\right) I_{z}^{i}+\hat{\mathcal{H}}_{r}, \quad 3 / 2 \tau_{w}<t<2 \tau_{w},
\end{array}\right.
\end{aligned}
$$

as

$$
\widehat{\widetilde{U}}_{0}(t)=\hat{U}_{n}^{\mathrm{CS}}(t) \hat{U}_{i}^{\mathrm{CS}}(t),
$$

with

$$
\begin{aligned}
& \hat{U}_{n}^{\mathrm{CS}}(t)=\exp \left(-i \int_{0}^{t} \widehat{\mathcal{H}}_{n}^{\mathrm{CS}}(\tau) d \tau\right), \\
& \hat{U}_{i}^{\mathrm{CS}}(t)=\exp \left(-i \int_{0}^{t}\left(\widehat{\widetilde{\mathcal{H}}}_{i}^{\mathrm{CS}}(\tau)+\hat{\mathcal{H}}_{r}\right) d \tau\right) .
\end{aligned}
$$

The dipolar interaction Hamiltonian from Eq. (31) has the form

$$
\widehat{\mathcal{H}}_{i}=\left(Z_{p}^{i j} \hat{F}_{p}+Z_{p}^{i j^{*}} \hat{F}_{p}^{\dagger}\right)\left(2 \hat{I}_{z}^{i} \hat{I}_{z}^{j}-1 / 2\left(\hat{I}_{i}^{+} \hat{I}_{j}^{-}+\hat{I}_{i}^{-} \hat{I}_{j}^{+}\right)\right) .
$$

The Hamiltonians $\widehat{\widetilde{\mathcal{H}}}_{n}^{\mathrm{CS}}(t)$ and $\widehat{\widetilde{\mathcal{H}}}_{i}^{\mathrm{CS}}(t)+\hat{\mathcal{H}}_{r}$ commute and so do $\hat{U}_{n}^{\mathrm{CS}}(t)$ and $\hat{U}_{i}^{\mathrm{CS}}(t)$. In addition, the $\widehat{\widetilde{\mathcal{H}}}_{i}^{\mathrm{CS}}(t)+\hat{\mathcal{H}}_{r}$ is block diagonal and can be diagonalized according to Refs. 33 and 36 with $(k=i, j)$,

$$
\begin{aligned}
& \sum_{p=1}^{2}\left(Z_{p}^{k} \hat{F}_{p}+Z_{p}^{k^{*}} \hat{F}_{p}^{\dagger}\right) \hat{I}_{z}^{k}+\hat{\mathcal{H}}_{r}=\hat{D}_{k} \hat{\mathcal{H}}_{r} \hat{D}_{k}^{-1}, \\
& \sum_{p=1}^{2}-\left(Z_{p}^{k} \hat{F}_{p}+Z_{p}^{k^{*}} \hat{F}_{p}^{\dagger}\right) \hat{I}_{z}^{k}+\hat{\mathcal{H}}_{r}=\hat{D}_{k}^{-1} \hat{\mathcal{H}}_{r} \hat{D}_{k}
\end{aligned}
$$

with

$$
\hat{D}_{k}=\exp \left\{-\sum_{p=1}^{2}\left(\frac{Z_{p}^{k}}{p \omega_{r}} \hat{F}_{p}-\frac{Z_{p}^{k^{*}}}{p \omega_{r}} \hat{F}_{p}^{\dagger}\right) \hat{I}_{z}^{k}\right\} .
$$

Insertion of these results into Eq. (A1), defining $\hat{D}=\hat{D}_{i} \hat{D}_{j}$ with $\left[\hat{D}_{i}, \hat{D}_{j}\right]=0$ and using $\left[\hat{U}_{n}^{\mathrm{CS}}(t), \hat{U}_{i}^{\mathrm{CS}}(t)\right]=0$ we get

$$
\widehat{\mathcal{H}}_{\mathrm{int}}(t)=\left\{\begin{array}{l}
\hat{U}_{n}^{\mathrm{CS}}(t) \hat{D} \hat{U}_{r}(t) \hat{D}^{-1} \hat{\mathcal{H}}_{1} \hat{D} \hat{U}_{r}^{-1}(t) \hat{D}^{-1} \hat{U}_{n}^{\mathrm{CS}-1}(t), \\
\hat{U}_{n}^{\mathrm{CS}-1}\left(t-\tau_{w}\right) \hat{D}^{-1} \hat{U}_{r}\left(t-\tau_{w}\right) \hat{D} \hat{\mathcal{H}}_{1} \hat{D}^{-1} \hat{U}_{r}^{-1}\left(t-\tau_{w}\right) \hat{D} \hat{U}_{n}^{\mathrm{CS}}\left(t-\tau_{w}\right), \\
\hat{U}_{n}^{\mathrm{CS}}\left(t-2 \tau_{w}\right) \hat{D} \hat{U}_{r}\left(t-2 \tau_{w}\right) \hat{D}^{-1} \hat{\mathcal{H}}_{1} \hat{D} \hat{U}_{r}^{-1}\left(t-2 \tau_{w}\right) \hat{D}^{-1} \hat{U}_{n}^{\mathrm{CS}}\left(t-2 \tau_{w}\right),
\end{array}\right.
$$

respectively, for the three time intervals, where we assumed that $n_{w}$ is even in $\tau_{w}=n_{w} \tau_{r}$, and $\hat{U}_{r}\left(1 / 2 \tau_{w}\right)=\hat{1}$. When we change the variables of integration and take into account that the Hamiltonians are constant during each time interval, the average Hamiltonian can be rewritten as

$$
\begin{aligned}
\widehat{\mathcal{H}}_{Q}= & \frac{1}{2 \tau_{w}} \int_{-1 / 2 \tau_{w}}^{1 / 2 \tau_{w}}\left\{\hat{U}_{n}^{\mathrm{CS}}(t) \hat{D} \hat{U}_{r}(t) \hat{D}^{-1} \hat{\mathcal{H}}_{1} \hat{D} \hat{U}_{r}^{-1}(t)\right. \\
& \times \hat{D}^{-1} \hat{U}_{n}^{\mathrm{CS}-1}(t)+\hat{U}_{n}^{\mathrm{CS}-1}(t) \hat{D}^{-1} \hat{U}_{r}(t) \\
& \left.\times \hat{D} \hat{\mathcal{H}}_{1} \hat{D}^{-1} \hat{U}_{r}^{-1}(t) \hat{D} \hat{U}_{n}^{\mathrm{CS}}(t)\right\} d t .
\end{aligned}
$$

To evaluate this integral we must consider the following computational steps: (a) calculation of $\hat{A}=\hat{D}^{-1} \hat{\mathcal{H}}_{1} \hat{D}$ and $\hat{A}^{\prime}=\hat{D} \hat{\mathcal{H}}_{1} \hat{D}^{-1}$ by expanding the diagonalization operators; (b) application of the rotor operator $\hat{A}(t)=\hat{U}_{r}(t) \hat{A} \hat{U}_{r}^{-1}$ and $\hat{A}^{\prime}(t)=\hat{U}_{r}(t) \hat{A}^{\prime} \hat{U}_{r}^{-1}(t)$; (c) transformation of $\hat{A}(t)$ and $\hat{A}^{\prime}(t) \quad$ to $\hat{B}(t)=\hat{D} \hat{A}(t) \hat{D}^{-1} \quad$ and $\hat{B}^{\prime}(t)=\hat{D}^{-1} \hat{A}^{\prime}(t) \hat{D}$; (d) and application of the isotropic chemical shift operator $\hat{C}(t)=\hat{U}_{n}^{\mathrm{CS}}(t) \hat{B}(t) \hat{U}_{n}^{\mathrm{CS}-1}$ and $\hat{C}^{\prime}(t)=\hat{U}_{n}^{\mathrm{CS}-1} \hat{B}^{\prime}(t) \hat{U}_{n}^{\mathrm{CS}}(t)$. To perform the first step we calculate the $D$-transformations of the terms of $\hat{\mathcal{H}}_{1}$ :

$$
\begin{aligned}
& \hat{D} \hat{F}_{p} \hat{I}_{z}^{i} \hat{I}_{z}^{j} \hat{D}^{-1}=\hat{F}_{p} \hat{I}_{z}^{i} \hat{I}_{z}^{j}, \\
& \hat{D}^{-1} \hat{F}_{p} \hat{I}_{z}^{i} \hat{I}_{z}^{j} \hat{D}^{-} \hat{F}_{p} \hat{I}_{z}^{i} \hat{I}_{z}^{j},
\end{aligned}
$$

$$
\begin{aligned}
& \hat{D} \hat{F}_{p} \hat{I}_{+}^{i} \hat{I}_{-}^{j} \hat{D}^{-1}=\hat{\Delta}^{i j} \hat{F}_{p} \hat{I}_{+}^{i} \hat{I}_{-}^{j}, \\
& \hat{D}^{-1} \hat{F}_{p} \hat{I}_{+}^{i} \hat{I}^{j} \hat{D}^{-}=\hat{\Delta}^{i j-1} \hat{F}_{p} \hat{I}_{+}^{i} \hat{I}_{-}^{j}
\end{aligned}
$$

with

$$
\Delta^{i j}=\exp \left\{-\sum_{p=1}^{2}\left(\frac{Z_{p}^{i}-Z_{p}^{j}}{p \omega_{r}}-\frac{Z_{p}^{i^{*}}-Z_{p}^{j^{*}}}{p \omega_{r}}\right)\right\}
$$

and $\hat{\Delta}^{i j}=\hat{\Delta}^{j i-1}$ and $\hat{\Delta}^{i j+}=\hat{\Delta}^{i j-1}$. This exponent can be expanded in a Fourier series using $Z_{p}^{i}-Z_{p}^{j}=\mid Z_{p}^{i}$ $-Z_{p}^{j} \mid \exp \left(i \phi_{p}^{i j}\right)$ :

$$
\begin{aligned}
\hat{\Delta}^{i j}= & \prod_{p=1}^{2} \sum_{m=0}^{\infty} \sum_{n=0}^{\infty} \frac{1}{n !}\left(-\frac{Z_{p}^{i}-Z_{p}^{j}}{p \omega_{r}} \hat{F}_{p}\right)^{n} \frac{1}{m !} \\
& \times\left(-\frac{Z_{p}^{i^{*}}-Z_{p}^{j^{*}}}{p \omega_{r}} \hat{F}_{-p}\right)^{m} \\
= & \prod_{p=1}^{2} \sum_{m=0}^{\infty} \sum_{l=-m}^{\infty} \frac{1}{m !} \frac{1}{(m+l) !} \\
& \times\left(-\frac{Z_{p}^{i}-Z_{p}^{j}}{p \omega_{r}}\right)^{m+l}\left(\frac{Z_{p}^{i^{*}}-Z_{p}^{j^{*}}}{p \omega_{r}}\right)^{m} \hat{F}_{l p} \\
= & \prod_{p=1}^{2} \sum_{m=0}^{\infty} \sum_{l=-\infty}^{\infty} \frac{1}{m !} \frac{(-1)^{m}}{\Gamma(m+l+1)}
\end{aligned}
$$




$$
\begin{aligned}
& \times\left(-\frac{\left|Z_{p}^{i}-Z_{p}^{j}\right|}{p \omega_{r}}\right)^{2 m+l} \exp \left(i l \phi_{p}^{i j}\right) \hat{F}_{l p} \\
= & \left.\sum_{k=-\infty}^{\infty} \sum_{l=-\infty}^{\infty} J_{k} \mid-2 \frac{\left|Z_{2}^{i}-Z_{2}^{j}\right|}{2 \omega_{r}}\right) J_{l} \\
& \times\left(-2 \frac{\left|Z_{l}^{i}-Z_{l}^{j}\right|}{\omega_{r}}\right) \exp \left(i l \phi_{1}^{i j}+i k \phi_{2}^{i j}\right) \hat{F}_{l+2 k} \\
= & \sum_{m=-\infty}^{\infty} K_{m}^{i j} \hat{F}_{m}
\end{aligned}
$$

with

$$
\begin{aligned}
K_{m}^{i j}= & \sum_{k=-\infty}^{\infty} J_{k}\left(-\frac{\left|Z_{2}^{i}-Z_{2}^{j}\right|}{\omega_{r}}\right) J_{m-2 k}\left(-2 \frac{\left|Z_{1}^{i}-Z_{1}^{j}\right|}{\omega_{r}}\right) \\
& \times \exp \left(i(m-2 k) \phi_{1}^{i j}+i k \phi_{2}^{i j}\right)
\end{aligned}
$$

and $J_{k}(x)$ the $k$ th order Bessel function of $x$. Thus in step (a) the transformation results in

$$
\begin{aligned}
\hat{A}= & \sum_{p=1}^{2}\left\{2\left(Z_{p}^{i j} F_{p}+Z_{p}^{i j^{*}} F_{p}^{\dagger}\right) I_{z}^{i} I_{z}^{j}-1 / 2\right. \\
& \times \sum_{m=-\infty}^{\infty}\left(\left(Z_{p}^{i j} K_{m}^{i j} F_{m+p}+Z_{p}^{i j^{*}} K_{m}^{i j} F_{m-p}\right) I_{i}^{+} I_{j}^{-}\right. \\
& \left.\left.+\left(Z_{p}^{i j} K_{-m}^{i j^{*}} F_{m+p}+Z_{p}^{i j^{*}} K_{-m}^{i j^{*}} F_{m-p}\right) I_{i}^{-} I_{j}^{+}\right)\right\}, \\
\hat{A}^{\prime}= & \sum_{p=1}^{2}\left\{2\left(Z_{p}^{i j} F_{p}+Z_{p}^{i j^{*}} F_{p}^{\dagger}\right) I_{z}^{i} I_{z}^{j}-1 / 2\right. \\
& \times \sum_{m=-\infty}^{\infty}\left(\left(Z_{p}^{i j} K_{-m}^{i j^{*}} F_{m+p}+Z_{p}^{i j^{*}} K_{-m}^{i j^{*}} F_{m-p}\right) I_{i}^{+} I_{j}^{-}\right. \\
& \left.\left.+\left(Z_{p}^{i j} K_{m}^{i j} F_{m+p}+Z_{p}^{i j^{*}} K_{m}^{i j} F_{m-p}\right) I_{i}^{-} I_{j}^{+}\right)\right\} .
\end{aligned}
$$

In the next step (b) we use the fact that

$$
\begin{aligned}
& U_{r}(t) F_{p} U_{r}^{-1}(t)=F_{p} \exp \left(-i p \omega_{r} t\right), \\
& U_{r}^{-1}(t) F_{p} U_{r}(t)=F_{p} \exp \left(i p \omega_{r} t\right)
\end{aligned}
$$

and replace all $F_{p}$ in Eq. (A15) by $F_{p} \exp \left(-i p \omega_{r}\right)$ and $F_{p} \exp \left(i p \omega_{r}\right)$ to obtain $A(t)$ and $A^{\prime}(t)$, respectively. In step (c) an additional $D$-transformation must be performed. The results for $B(t)$ and $B^{\prime}(t)$, obtained again by using Eqs. (11)-(13), are cumbersome and are therefore not shown here. Their coefficients of $I_{z}^{i} I_{z}^{j}$ and $I_{ \pm}^{i} I_{ \pm}^{j}$ contain multiples of the $K$-parameters. In the last step the isotropic chemical shift Hamiltonian is applied to the bilinear spin operators

$$
\begin{aligned}
& U_{n}^{\mathrm{CS} \pm 1}(t) I_{z}^{i} I_{z}^{j} U_{n}^{\mathrm{CS} \mp 1}=I_{z}^{i} I_{z}^{j}, \\
& U_{n}^{\mathrm{CS} \pm 1}(t) I_{+}^{i} I_{-}^{j} U_{n}^{\mathrm{CS} \mp 1}=I_{+}^{i} I_{-}^{j} \exp \left(\mp\left(\Delta \omega^{i}-\Delta \omega^{j}\right) t\right), \\
& U_{n}^{\mathrm{CS} \pm 1}(t) I_{-}^{i} I_{+}^{j} U_{n}^{\mathrm{CS} \mp 1}=I_{-}^{i} I_{+}^{j} \exp \left( \pm\left(\Delta \omega^{i}-\Delta \omega^{j}\right) t\right)
\end{aligned}
$$

in order to obtain expressions for $C(t)$ and $C^{\prime}(t)$ from $B(t)$ and $B^{\prime}(t)$. Insertion of the results of these calculations in Eq. (A10) yields for the zero order average Hamiltonian the following:

$$
\begin{aligned}
\widehat{\mathcal{H}}_{Q}= & -\frac{1}{4 \tau_{w}} \sum_{i<j} \sum_{p=-2}^{2} Z_{p}^{i j} \sum_{k, n=-\infty}^{\infty}\left(K_{k}^{i j} K_{n+p}^{i j^{*}} \hat{F}_{k-n} \hat{I}_{+}^{i} \hat{I}_{-}^{j}\right. \\
& \times \int_{-\frac{1}{2} \tau_{w}}^{\frac{1}{2} \tau_{w}} \exp \left(i\left(\Delta \bar{\omega}^{i}-\Delta \bar{\omega}^{j}-n \omega_{r}\right) t\right) d t \\
& +K_{-k}^{i j^{*}} K_{n-p}^{i j} \hat{F}_{k+n} \hat{I}_{+}^{j} \hat{I}_{-}^{i} \int_{-\frac{1}{2} \tau_{w}}^{\frac{1}{2} \tau_{w}} \exp \left(i \left(\Delta \bar{\omega}^{j}-\Delta \bar{\omega}^{i}\right.\right. \\
& \left.\left.+n \omega_{r}\right) t\right) d t+K_{-k}^{i j *} K_{n-p}^{i j} \hat{F}_{k+n} \hat{I}_{+}^{i} \hat{I}_{-}^{j} \int_{-\frac{1}{2} \tau_{w}}^{\frac{1}{2} \tau_{w}} \exp \left(i \left(\Delta \bar{\omega}^{j}\right.\right. \\
& \left.\left.-\Delta \bar{\omega}^{i}+n \omega_{r}\right) t\right) d t+K_{k}^{i j} K_{n+p}^{i j *} \hat{F}_{k-n} \hat{I}_{+}^{j} \hat{I}_{-}^{i} \int_{-\frac{1}{2} \tau_{w}}^{\frac{1}{2} \tau_{w}} \\
& \left.\times \exp \left(i\left(\Delta \bar{\omega}^{i}-\Delta \bar{\omega}^{j}-n \omega_{r}\right) t\right) d t\right)
\end{aligned}
$$

and the actual integration gives Eqs. (52) and (53). This Hamiltonian has only a $\left(I_{x}^{i} I_{x}^{j}+I_{y}^{i} I_{y}^{j}\right)=1 / 2\left(I_{+}^{i} I_{-}^{j}+I_{-}^{i} I_{+}^{j}\right)$ term, since the integral of the $I_{z}^{i} I_{z}^{j}$ coefficient, containing only terms of exponents $\exp \left( \pm i p \omega_{r} t\right)$ is zero when $\tau_{w}$ is a multiple of the rotor period $\tau_{r}$.

${ }^{1}$ B. J. van Rossum et al., Spectrochim. Acta A 54, 1167 (1998).

${ }^{2}$ B. J. van Rossum, S. Prytulla, H. Oschkinat, and H. J. M. de Groot, Proceedings of the Joint 29th AMPERE-13th ISMAR International Conference, Berlin, Vol. 1, p. 38, 1998.

${ }^{3}$ R. Tycko and G. Dabbagh, Chem. Phys. Lett. 173, 461 (1990).

${ }^{4}$ R. G. Griffin, Nat. Struct. Biol. 5, 508 (1998).

${ }^{5}$ A. E. Bennet, J. H. Ok, R. G. Griffin, and S. Vega, J. Chem. Phys. 96, 8624 (1992).

${ }^{6}$ M. Baldus, M. Tomaselli, B. H. Meier, and R. R. Ernst, Chem. Phys. Lett. 230, 329 (1994).

${ }^{7}$ J. M. Joers, R. Rosanske, T. Gullion, and J. R. Garbow, J. Magn. Reson. 106, 123 (1994)

${ }^{8}$ B. Q. Sun et al., J. Chem. Phys. 102, 702 (1995).

${ }^{9}$ Y. K. Lee et al., Chem. Phys. Lett. 242, 304 (1995).

${ }^{10}$ D. M. Gregory, D. J. Mitchell, and G. P. Drobny, Chem. Phys. Lett. 246, 654 (1995).

${ }^{11}$ R. Verel, M. Baldus, M. Ernst, and B. H. Meier, Chem. Phys. Lett. 287, 421 (1998).

${ }^{12}$ I. J. Lowe, Phys. Rev. Lett. 2, 285 (1959).

${ }^{13}$ E. R. Andrew, A. O. Bradbury, and R. G. Eades, Nature (London) 182, 1659 (1958)

${ }^{14}$ G. J. Boender et al., Chem. Phys. Lett. 237, 502 (1995).

${ }^{15}$ B. J. van Rossum, H. Förster, and H. J. M. de Groot, J. Magn. Reson. 124, 516 (1997).

${ }^{16}$ T. S. Balaban et al., Biochemistry 34, 15259 (1995).

${ }^{17}$ G. J. Boender, S. Vega, and H. J. M. de Groot, Mol. Phys. 95, 921 (1998).

${ }^{18}$ J. H. Shirley, Phys. Rev. 138, 979 (1965).

${ }^{19}$ G. J. Boender, thesis, Leiden University, 1996.

${ }^{20}$ M. M. Maricq and J. S. Waugh, J. Chem. Phys. 70, 3300 (1979).

${ }^{21}$ C. Cohen Tannoudji, B. Diu, and F. Laloe, Quantum Mechanics (Hermann, Paris, 1977).

${ }^{22}$ Quantum Mechanics, 3rd ed., Course of Theoretical Physics, edited by L. D. Landau and E. M. Lifshitz (Oxford, Pergamon, New York, 1981).

${ }^{23}$ M. Mehring, Principles of High Resolution NMR Spectroscopy in Solids (Springer-Verlag, Berlin, 1983), 2nd Ed.

${ }^{24}$ G. Racah, Phys. Rev. 62, 438 (1942).

${ }^{25} \mathrm{U}$. Haeberlen, High Resolution NMR in Solids: Selective Averaging, Suppl. 1 Adv. Magn. Reson. (Academic, New York, 1976). 
${ }^{26}$ A. E. Bennett et al., J. Chem. Phys. 108, 9463 (1998).

${ }^{27}$ J. S. Waugh, L. M. Huber, and U. Haeberlen, Phys. Rev. Lett. 20, 180 (1968).

${ }^{28}$ J. S. Waugh, C. H. Wang, L. M. Huber, and R. L. Vold, J. Chem. Phys. 48, 662 (1968).

${ }^{29}$ R. R. Ernst, G. Bodenhausen, and A. Wokaun, Principles of Nuclear Magnetic Resonance in One and Two Dimensions (Clarendon, Oxford, 1987).

${ }^{30}$ O. Weintraub, S. Vega, Ch. Hoelger, and H. H. Limbach, J. Magn. Reson.,
Ser. A 109, 14 (1994).

${ }^{31}$ D. K. Sodikson, M. H. Levitt, S. Vega, and R. G. Griffin, J. Chem. Phys. 98, 6742 (1993)

${ }^{32}$ B. Q. Sun et al., J. Chem. Phys. 102, 702 (1995).

${ }^{33}$ S. Vega, J. Chem. Phys. 68, 5518 (1978).

${ }^{34}$ A. Wokaun and R. R. Ernst, J. Chem. Phys. 67, 1752 (1977).

${ }^{35}$ G. Goobes, G. J. Boender, and S. Vega (in preparation).

${ }^{36}$ D. B. Zax, G. Goelman, D. Abromovich, and S. Vega, Adv. Magn. Reson. 15, 219 (1990). 